\title{
Surface acoustic wave distribution and acousto-optic interaction in proton exchanged $\mathrm{LiNbO}_{3}$ waveguides
}

\author{
P ANNACHELVI, A SELVARAJAN and G V ANAND \\ Department of Electrical Communication Engineering, Indian Institute of Science, Bangalore \\ 560012 , India \\ MS received 17 February 1993; revised 16 July 1993
}

\begin{abstract}
The efficiency of acoustooptic (AO) interaction in YZ-cut proton exchanged (PE) $\mathrm{LiNbO}_{3}$ waveguides is theoretically analysed by determining the overlap between the optical and acoustic field distributions. The present analysis takes into account the perturbed SAW field distribution due to the presence of the $\mathrm{PE}$ layer on the $\mathrm{LiNbO}_{3}$ substrate determined by the rigorous layered medium approach. The overlap is found to be significant upto very high acoustic frequencies of the order of $5 \mathrm{GHz}$, whereas in the earlier analysis by vonHelmolt and Schaffer [6] for diffused waveguides, it was shown that the overlap integral rolls down to nearly zero at this high frequency range.
\end{abstract}

Keywords. Acoustooptic interaction; $\mathrm{LiNbO}_{3}$ waveguides; proton exchange.

PACS No. $\mathbf{4 2 \cdot 8 2}$

\section{Introduction}

Integrated optical devices based on proton exchanged $\mathrm{LiNbO}_{3}\left(\mathrm{PE}: \mathrm{LiNbO}_{3}\right)$ waveguides are being researched for various applications including signal processing, communication, etc. The devices based on the acoustooptic (AO) interaction in these waveguides are promising and have given rise to considerable activity in recent years. Technologically, proton exchange is a simpler and low temperature process, which is easy to perform. Immersion of $\mathrm{LiNbO}_{3}$ in proton sources such as benzoic acid leads to a high index region of $\mathrm{H}_{x} \mathrm{Li}_{1-x} \mathrm{NbO}_{3}$, with $77 \%$ of $\mathrm{Li}^{+}$replaced by protons [1]. The main advantages of PE waveguides are the large increase in the extraordinary refractive index, the nearly step index profile, and the high optical damage threshold [2]. Many AO experiments have been performed and devices realized [3,4], taking advantage of the special optical and acoustic properties of these waveguides. Yet, a rigorous computation of the basic $\mathrm{AO}$ interaction in these waveguides has not been reported so far. In this paper, we present a theoretical analysis of the $\mathrm{AO}$ interaction in PE waveguides by rigorously calculating the perturbed SAW distribution and the optical field distribution in the $\mathrm{PE}: \mathrm{LiNbO}_{3}$. The analysis leading to the determination of SAW distribution in $\mathrm{PE}: \mathrm{LiNbO}_{3}$ is based on a recent work [5], wherein the material constants are determined for the PE medium. 


\section{Calculation of the SAW field}

The Rayleigh wave propagation in a piezoelectric medium in governed by a set of four coupled differential equations [5]:

$$
\begin{aligned}
\rho \frac{\partial^{2} u_{j}}{\partial t^{2}}-C_{i j k l}^{\prime} \frac{\partial^{2} u_{k}}{\partial x_{i} \partial x_{l}}-e_{k i j}^{\prime} \frac{\partial^{2} \phi}{\partial x_{i} \partial x_{k}}=0, & i, j, k, l=1,2,3, \\
e_{i k l}^{\prime} \frac{\partial^{2} u_{k}}{\partial x_{i} \partial x_{l}}-\varepsilon_{i k}^{\prime} \frac{\partial^{2} \phi}{\partial x_{i} \partial x_{k}}=0, & i, k, l=1,2,3
\end{aligned}
$$

where $u_{i}$ is the $i$ th component of mechanical displacement, $\phi$ is the electric potential, $C_{i j k l}^{\prime}, e_{i k l}^{\prime}$ and $\varepsilon_{i k}^{\prime}$ are the rotated components of the elastic stiffness, piezoelectric and dielectric permittivity tensors respectively, and summation over repeated indices is implied. Using the coordinate system shown in figure 1 , solutions of (1) and (2) can be written as

$$
\begin{aligned}
& u_{i}=a_{i} \exp \left(j k \alpha x_{3}\right) \exp \left[j k\left(x_{1}-v t\right)\right], \quad i=1,2,3 \\
& \phi=a_{4} \exp \left(j k \alpha x_{3}\right) \exp \left[j k\left(x_{1}-v t\right)\right],
\end{aligned}
$$

where $a_{i}$ are the amplitude coefficients, $\alpha$ is the propagation constant in the $x_{3}$ direction, and $v$ is the SAW phase velocity. Substitution of (3) and (4) into (1) and (2) leads to the linear homogeneous matrix equation:

$$
\mathbf{M A}=0,
$$

where $M$ is a $4 \times 4$ matrix with elements involving the material constants and $\alpha$, and $A$ is a 4-dimensional column vector with elements $a_{i}, i=1, \ldots, 4$. Nontrivial solutions of (5) exist if and only if

$$
|\mathbf{M}|=0
$$

where the determinant $|\mathbf{M}|$ is a polynomial in $\alpha$ of degree eight. For a given phase velocity, (6) is solved to get the eight propagation constants $\alpha_{n}$ and the corresponding amplitude coefficient vectors $\mathbf{A}_{n}$. The procedure has to be carried out for both the substrate and the layer. Since the field components of Rayleigh waves must decay exponentially into the substrate, only four propagation constants with negative imaginary parts may be used for the substrate. For the layer all the eight eigenvalues are allowed. Thus, superposition of the partial wave solutions and denoting the
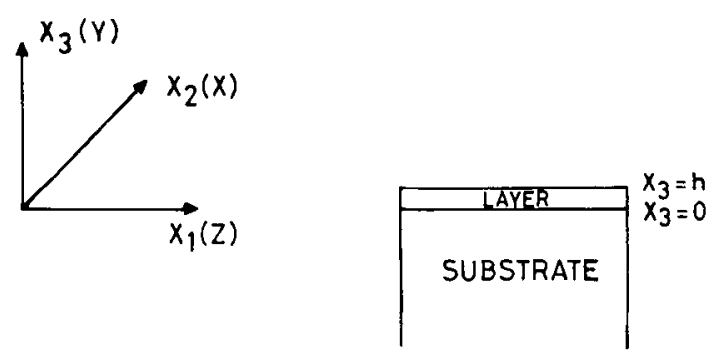

Figure 1. Schematic geometry of $\mathrm{PE}: \mathrm{LiNbO}_{3} . X, Y, Z$ are the crystal axes. 
potential by $u_{4}$ leads to the following solution for the Rayleigh wave in vector form:

$$
\mathbf{u}=\sum_{n} \mathbf{B}_{n} \mathbf{A}_{n} \exp \left(j k \alpha_{n} x_{3}\right) \exp \left[j k\left(x_{1}-v t\right)\right]
$$

where $\mathbf{u}$ is a 4-dimensional column vector with elements $u_{i}, \mathbf{A}_{n}$ are 4-dimensional column vectors with elements $a_{i n}$ and $B_{n}$ are the scalar quantities denoting partial wave amplitudes, with $n=1, \ldots, 4$ for the substrate and $n=5, \ldots, 12$ for the layer.

Formulation of the boundary conditions yields a linear homogeneous system of twelve equations, from which $B_{n}$ can be found. The boundary conditions are:

(i) At the layer-substrate interface $x_{3}=0$, the mechanical displacement components $u_{1}, u_{2}$ and $u_{3}$, the electric potential $\phi$, the stress components $T_{13}, T_{23}$ and $T_{33}$ and the electric displacement $D_{3}$ are continuous. The stress components $T_{i 3}$ and the electric displacement $D_{3}$ are given by,

$$
\begin{aligned}
& T_{i 3}=C_{i 3 k l}^{\prime} S_{k l}+e_{i 3 k}^{\prime} E_{k}, \quad i=1,2,3 \\
& D_{3}=e_{3 k l}^{\prime} S_{k l}-\varepsilon_{3 k}^{\prime} E_{k}
\end{aligned}
$$

(ii) At the free surface $x_{3}=h$, the stress components $T_{13}, T_{23}$ and $T_{33}$ vanish and the electric displacement $D_{3}$ is continuous. The twelve boundary conditions lead to the homogeneous equation,

$$
\mathbf{R B}=0 \text {, }
$$

where $\mathbf{R}$ is the boundary condition matrix of order $12 \times 12$, and $\mathbf{B}$ is the 12 dimensional column vector containing the 12 unknown partial wave amplitudes $B_{n}$.

For nontrival solutions of $(10)$, the boundary condition determinant $|\mathbf{R}|$ should vanish, and this can be accomplished by variation of the only free parameter, $k h$. For a given phase velocity, the boundary condition determinant determines the parameter $k h$ and this parameter describes the dispersive behaviour of SAW in the layered medium.

\section{Application to $\mathrm{PE}: \mathrm{LiNbO}_{3}$}

\subsection{Crystalline model}

The assumptions which we have adopted in the case of PE waveguides, following the earlier investigations [5], are:

(i) The PE region behaves like a homogeneous nonpiezoelectric medium.

(ii) For the exchange rate considered here, viz., $x \approx 0.7$, the crystalline structure of the $\mathrm{PE}$ medium $\left(\mathrm{H}_{x} \mathrm{Li}_{1-x} \mathrm{NbO}_{3}\right)$ can be taken as trigonal.

(iii) For the PE medium, $\varepsilon_{11}=\varepsilon_{22}=\varepsilon_{33}=55 \varepsilon_{0}$, and $\rho=4500 \mathrm{kgm}^{-3}$.

(iv) The set of material constants and the dispersion curve corresponding to the YZ-configuration are reliable for waveguides fabricated in diluted melts containing 0.25 to $2 \mathrm{~mol} \%$ of lithium benzoate.

\subsection{SAW field distribution}

The relevant material constants $C_{i j k l}, e_{i j k}$ and $\varepsilon_{i j}$ of $\mathrm{LiNbO}_{3}, v_{\mathrm{SAw}}$ and the Euler angles used to find the rotated components, $C_{i j k i}^{\prime}, e_{i j k}^{\prime}$ and $\varepsilon_{i j}^{\prime}$ are taken from [6] and 


\section{$P$ Annachelvi, A Selvarajan and $G V$ Anand}

[7]. To check the correctness of matrix $\mathbf{M}$ in (5), $x$ and $\mathbf{A}$ are calculated for the case of SAW in bulk $\mathrm{LiNbO}_{3}$. The results agree well with the values reported by vonHelmholt and Schaffer [6]. For the PE layer, which is nonpiezoelectric, $e=0$ and the other constants of the PE medium $C_{i j k l}$ and $\varepsilon_{i j}$ and the dispersion curve are taken from [5].

The scalar constants $x_{n}$ and vectors $\mathrm{A}_{n}$ for a given $v_{\mathrm{SAW}}$ are determined for the substrate and the layer individually from (6). For different values of $v_{\mathrm{SAw}}$, different sets of $x_{n}$ and $A_{n}$ are obtained. The value of $k h$ corresponding to each $v_{\text {SAw }}$ is taken from the dispersion curve in the calculation of $B_{n}$. In finding $B_{n}$, the 12 boundary condition equations reduce to 8 equations because of the following reasons:

(i) In the nonpiezoelectric layer and the piezoelectric substrate combination, the continuity of $\phi$ at the layer-substrate interface and the continuity of $D_{3}$ at the free surface are satisfied by taking the potential in the form given below [8]:

$$
\phi=\phi_{0} \exp \left(-i k^{\prime \prime} x_{3}\right)\left(\frac{\exp \left(k^{\prime} x_{3}\right)+B \exp \left(-k^{\prime} x_{3}\right)}{1+B}\right),
$$

where

$$
\begin{aligned}
& k^{\prime}=k\left(\frac{\varepsilon_{11} \varepsilon_{33}-\varepsilon_{13}^{2}}{\varepsilon_{33}^{2}}\right)^{1 / 2}, \\
& k^{\prime \prime}=k \frac{\varepsilon_{13}}{\varepsilon_{33}} \\
& B=\left(\frac{k^{\prime} \varepsilon_{33}+k \varepsilon_{0}}{k^{\prime} \varepsilon_{33}-k \varepsilon_{0}}\right) \exp \left(2 k^{\prime} h\right),
\end{aligned}
$$

$\phi$ denotes the potential in the layer and $\phi_{0}$ denotes the substrate potential evaluated at $x_{3}=0$. For the present case of PE medium, $k^{\prime \prime}=0 ; k^{\prime}=k ; B=\left(\varepsilon_{33}+\varepsilon_{0}\right) /\left(\varepsilon_{33}-\varepsilon_{0}\right)$ $\exp (2 k h)$.

(ii) In the YZ-cut trigonal crystals, the sagittal plane $\left(X_{1}, X_{3}\right.$ plane in figure 1$)$ is

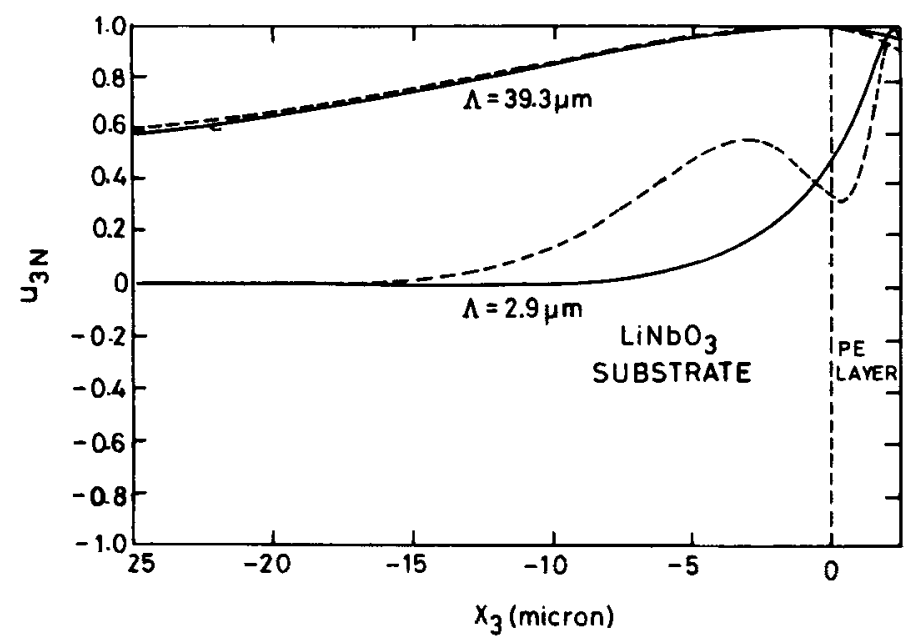

Figure 2. Normalized mechanical displacement distribution, $u_{3 N}$ in $\mathrm{PE}: \mathrm{LiNbO}_{3}$ through the present analysis (---) and in bulk $\mathrm{LiNbO}_{3}$ using vonHelmolt't analysis $(\longrightarrow)$; waveguide depth $=2.5 \mu \mathrm{m}$. 
the mirror plane for elastic symmetry and therefore the mechanical displacement component $u_{2}=0$ and all the associated boundary condition matrix elements vanish.

Thus, in the case of the substrate, the solutions $u_{1}, u_{3}$ and $\phi$ contain linear combinations of four partial wave solutions with appropriate coefficients $\alpha_{n}$ and $a_{n}$. In the guiding layer, $u_{1}$ and $u_{3}$ are given by linear combinations of four partial wave solutions and $\phi$ is given by (11). The partial field amplitudes are normalized to a SAW power flow $P_{1 m}=1 \mathrm{~W} / \mathrm{m}$ and $\omega=1 \mathrm{rad} / \mathrm{s}$. For very small values of $k h$ (i.e., $\Lambda \gg h$ ), it is seen from figure 2 that the perturbation due to the presence of the PE layer is negligible and the SAW distribution resembles that in the bulk $\mathrm{LiNbO}_{3}$ medium. The case of $\mathrm{SAW}$ in bulk $\mathrm{LiNbO}_{3}$ is treated in the earlier analysis by vonHelmolt and Schaffer [6].

The strain component $S_{1}$ and the electric field component $E_{1}$ are continuous across the layer-substrate interface. The component $S_{3}$, which is along the depth direction, has different magnitudes at the interface owing to the different decay coefficients of the substrate and the layer media.

\section{Optical field distribution}

The steplike refractive index profile of the proton exchanged waveguides is considered. This index profile leads to the optical field for $\mathrm{TE}_{0}$ mode given by [9],

$$
\begin{array}{ll}
U_{m}\left(x_{3}\right)=\cos \left[\kappa_{f}\left(-x_{3}+h\right)\right]+\frac{\kappa_{c}}{\kappa_{f}} \sin \left[\kappa_{f}\left(-x_{3}+h\right)\right] & 0 \leqslant x_{3} \leqslant h \\
U_{m}\left(x_{3}\right)=\left[\cos \left(\kappa_{f} h\right)+\frac{\kappa_{c}}{\kappa_{f}} \sin \left(\kappa_{f} h\right)\right] \exp \left(\kappa_{s} x_{3}\right) & x_{3} \leqslant 0
\end{array}
$$

where $\kappa_{f}, \kappa_{c}$ and $\kappa_{s}$ are the propagation constants in the film, cover and the substrate regions respectively and $h$ is the waveguide depth.

\section{Theory of guided wave acoustooptic interaction}

In our geometry, the co-ordinate axes $\left(x_{1}, x_{2}, x_{3}\right)$ coincide with the crystal axes $(Z, X, Y)$. The acoustooptic diffraction efficiency of an optical guided wave perfectly phase matched to the SAW for the isotropic case, with incident and diffracted optical modes being the same with respect to polarization, is given by the well known formula [10]:

$$
\eta=\sin ^{2}\left(\frac{\pi n_{\mathrm{eff}}^{2} L}{2 \lambda \cos \theta_{B}}\left|\Delta B_{\max }\right||\Gamma|\right)
$$

where, $n_{\text {eff }}$ is the effective modal refractive index, $\lambda$ is the free space optical wavelength, $\Gamma$ is the coupling function or the overlap integral, $L$ is the interaction length or acoustic aperture and $\theta_{B}$ is the Bragg angle. The AO diffraction efficiency strongly depends on the overlap $\Gamma$ between the optical and acoustic fields. $\Gamma$ which depends solely on the waveguide parameters and the acoustic frequency is given by,

$$
|\Gamma|=\left|\frac{\int_{-\infty}^{h}\left|U_{m}\right|^{2} U_{a i j} \mathrm{~d} x_{3}}{\int_{-\infty}^{h}\left|U_{m}\right|^{2} \mathrm{~d} x_{3}}\right|,
$$


where,

$$
\begin{aligned}
U_{a i j} & =\frac{\Delta B_{i j}}{\left|\Delta B_{i j \max }\right|}, \\
\Delta B_{i j} & =p_{i j k m}^{E} S_{k m}+r_{i j k}^{S} E_{k} \quad i, j, k, m=1,2,3
\end{aligned}
$$

where $\Delta B_{i j}$ is the change in the optical indicatrix created by SAW, $U_{m}\left(x_{3}\right)$ is the optical mode profile of the waveguide, $p_{i j k m}^{E}$ is the strain optic tensor at constant $E, S_{k m}$ are the strain components of SAW, $r_{i j k}^{S}$ is the electrooptic tensor at constant $S$ and $E_{k}$ are the electric field components of SAW.

\section{Interaction in proton exchanged waveguide}

The optical mode field as in (12) and the SAW field distribution as in (7) corresponding to the proton exchanged waveguides are used in (14) to determine the overlap integral. The integral is evaluated in the two regions (layer and substrate) using the appropriate functions, $U_{a}$ and $U_{m}$. With the SAW propagating along $x_{1}$ and the TE polarized light along $x_{2}$, we have

$$
\Delta B_{1}=p_{33} S_{1}+p_{31} S_{3}+r_{33} E_{1} .
$$

The coefficients $p$ and $r$ are referred to the crystal axes, $X=1, Y=2, Z=3 . r_{33}$ in the $\mathrm{PE}$ region is $22.6 \times 10^{-12} \mathrm{~m} / \mathrm{V}$ [11] whereas, $r_{33}$ in the $\mathrm{LiNbO}_{3}$ substrate is $30.8 \times 10^{-12} \mathrm{~m} / \mathrm{V}$. The proton exchanged waveguides considered in the present work are the annealed proton exchanged waveguides. The annealing process may involve migration of $\mathrm{Li}$ ions from adjacent regions of the substrate into the waveguiding region in addition to the removal of hydrogen bonded $\mathrm{OH}$ and the diffusion of free $\mathrm{OH}$ into the substrate. In this situation the distorted unit cell structure in the waveguide may tend to change back to that of virgin $\mathrm{LiNbO}_{3}$. As a consequence, the electrooptic effect would be restored and propagation losses reduced [12].

The values of $p_{33}$ and $p_{31}$ for $\mathrm{LiNbO}_{3}$ are 0.071 and 0.179 respectively. For the $\mathrm{PE}$ region, values of $p_{33}$ and $p_{31}$ are assumed to be the same as those in $\mathrm{LiNbO}_{3}$. The optical wavelength $\lambda$ is taken to be $0.83 \mu \mathrm{m}$ and the value of $\Delta n$ is chosen for a given waveguide depth such that the waveguide operates in the single mode region (for $d=1 \cdot 33, \Delta n=0.01$ ). Since the substrate is piezoelectric, the contribution to $\Delta B$ is more due to the electrooptic effect (EO) than due to the acoustooptic effect (AO) in this region. In the nonpiezoelectric layer, the $\mathrm{AO}$ contribution dominates. The AO and $\mathrm{EO}$ contributions to $\Delta B$ are plotted in figure 3.

The overlap integral is plotted as a function of acoustic frequency for different waveguide parameters in figures 4 and 5 . At very low and very high acoustic frequencies, when the waveguide depth $h$ is not comparable to the acoustic wavelength $\Lambda$, the overlap integral decreases with increasing acoustic frequency. It is also seen that at high acoustic frequencies the overlap decreases with increasing waveguide depth. This is due to the fact that the penetration depth of SAW is inversely proportional to the acoustic frequency. The acoustic penetration depth (the depth at which the SAW amplitude is equal to $1 / e$ times its surface value) is plotted as a function of acoustic frequency in figure 6. It is clear from figures 4 and 5 that, depending upon the waveguide depth, the frequency range of interest can be obtained. Secondly, for a given waveguide depth, when the optical penetration depth is increased, i.e., when the optical field becomes less concentrated in the waveguide, the overlap is less compared to the case of waveguides with smaller penetration depth. The optical 


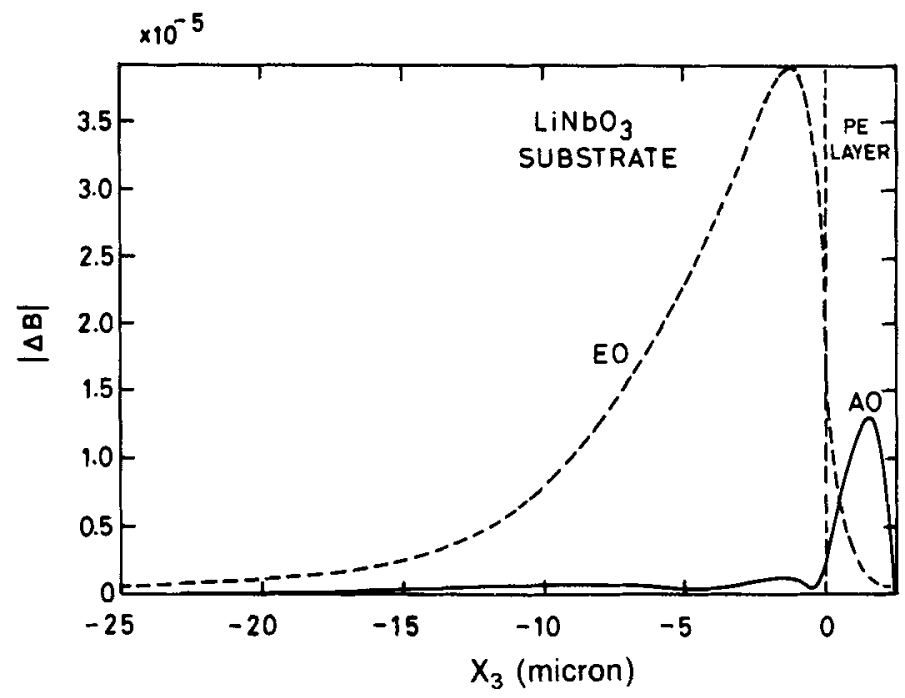

Figure 3. Normalized change in indicatrix $\Delta B$ due to electrooptic and acoustooptic effects; SAW frequency $=950 \mathrm{MHz}$.

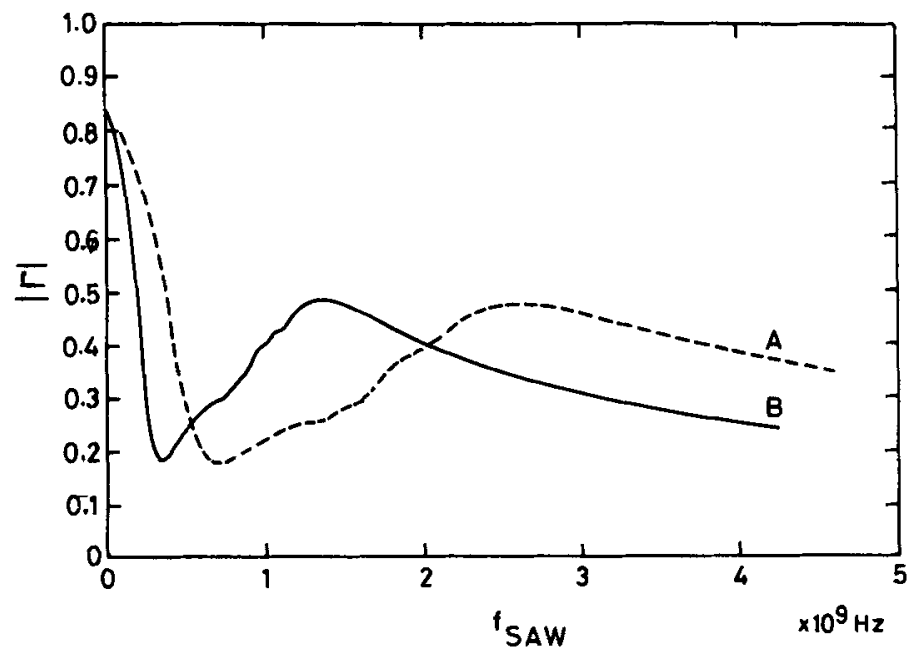

Figure 4. Overlap integral as a function of acoustic frequency; waveguide depth = $1.33 \mu \mathrm{m}$ for curve A and $2.5 \mu \mathrm{m}$ for curve $B$.

penetration depth is defined as the depth at which the optical field intensity is equal to $1 / e^{2}$ times the maximum intensity. For a fixed waveguide depth, the different optical penetration depths may be obtained by changing $\Delta n$.

When $h$ is comparable to $\Lambda$, there is no longer a monotonous decrease in the overlap with increase in frequency, but instead the overlap begins to increase as the frequency increases. In this intermediate frequency range, with $h$ being comparable to $\Lambda$, the layer offers a characteristic dimension to the system and there is a considerable contribution to $\Delta B$ from both the layer and the substrate. The proximity of the maximum of $\Delta B$ in the substrate to the layer-substrate interface leads to the increase in the overlap with increase in frequency. Because the substrate is piezoelectric and the layer is nonpiezoelectric, the maximum of $\Delta B$ lies in the substrate region. 
$P$ Annachelvi, $A$ Selvarajan and $G V$ Anand

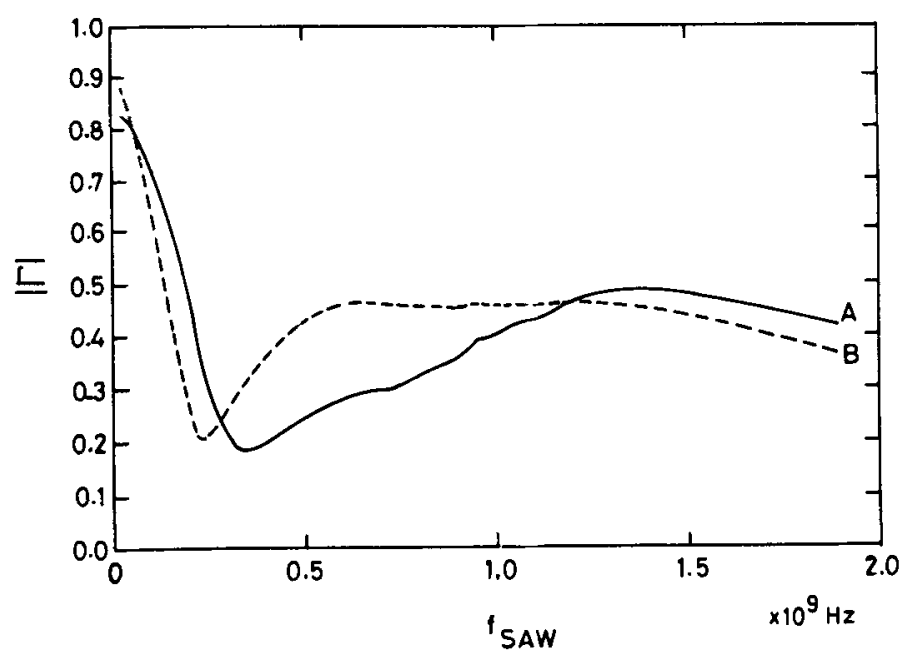

Figure 5. Overlap integral as a function of acoustic frequency for a given waveguide depth of $2.5 \mu \mathrm{m}$. The optical penetration depths are $6 \mu \mathrm{m}$ (curve A) and $9 \mu \mathrm{m}$ (curve B).

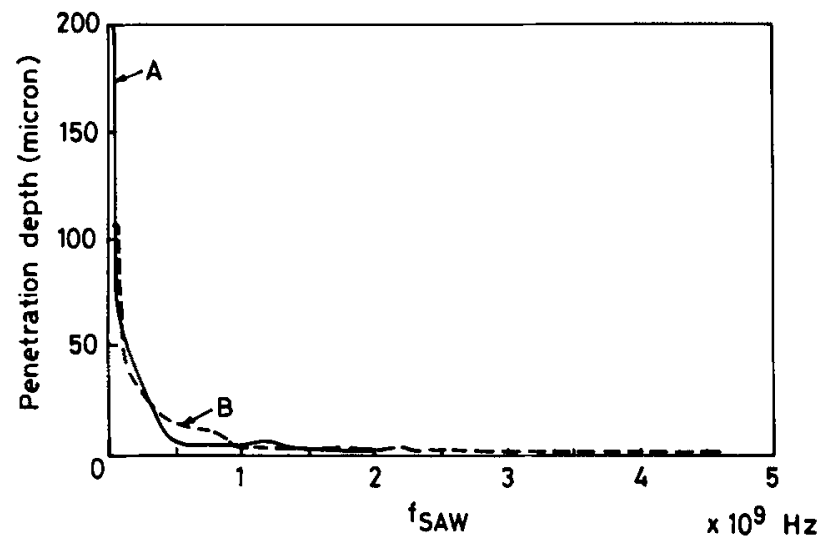

Figure 6. Acoustic penetration depth as a function of acoustic frequency for waveguide depth of A) $2.5 \mu \mathrm{m}$ and B) $1.33 \mu \mathrm{m}$.

The earlier analysis by vonHelmolt and Schaffer [6] is also carried out for the present case of the PE waveguides, the step index profile of the waveguides being taken into account in determining the optical field distribution. The result is shown in figure 7 along with that of the present improved analysis of the PE waveguides. For the present case, since the maximum of $\Delta B$ lies in the substrate region, the overlap integral is always less than 1 . In the earlier analysis, on the other hand, the maximum of $\Delta B$ is at, or, near the surface without an intermediate minimum and this leads to the overlap integral value of 1 at very low frequencies. The overlap is more at high frequencies in the present case. 


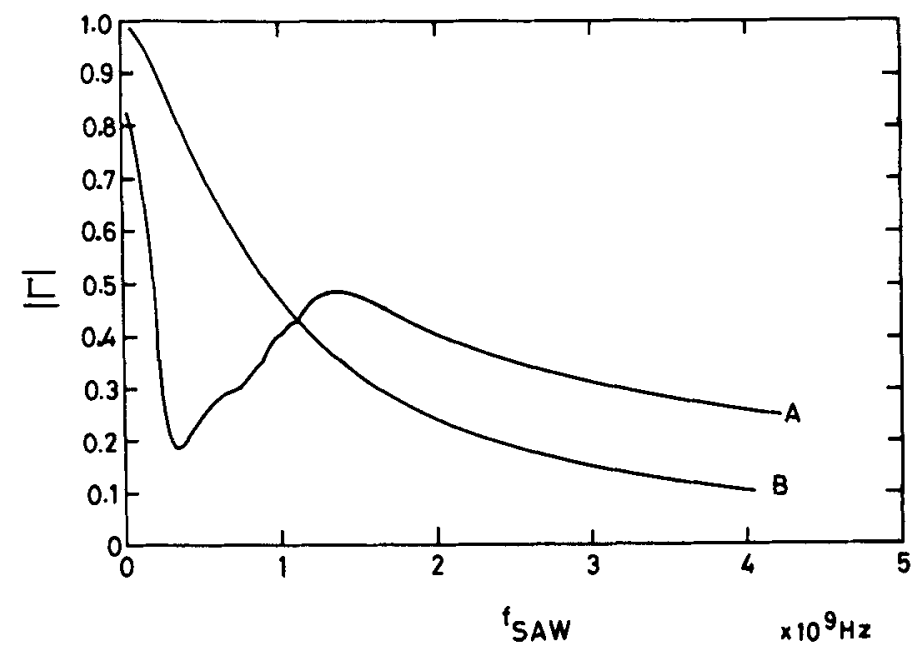

Figure 7. Frequency response of the overlap integral in the present case (curve $A$ ) and according to vonHelmolt's analysis (curve B).

\section{Conclusion}

The AO interaction in $\mathrm{PE}: \mathrm{LiNbO}_{3}$ is theoretically investigated using the improved calculation of SAW in these waveguides. The analysis presented here is more accurate since it takes into account the material constants of the PE medium and the dispersive behaviour of the waveguide. By the use of the modified SAW distribution and the improved calculation of the $\mathrm{AO}$ interaction, presented in this analysis, sophisticated design of guided SAW and AO devices based on $\mathrm{LiNbO}_{3}$ can be achieved.

\section{References}

[1] J L Jackel, C E Rice and J J Veselka, Appl. Phys. Lett. 41, 607 (1982)

[2] R A Becker, Appl. Phys. Lett. 43, 131 (1983)

[3] M Miyawaki and S Ogura, Appl. Phys. Lett. 47, 918 (1985)

[4] M Varasi, A Vannucci and S Reid, Proc. SPIE 1151, 457 (1989)

[5] E M Biebl and P Russer, IEEE Trans. Ultrason., Ferroelect. Freq. Control. 39, 330(1992)

[6] C H vonHelmolt and C Schaffer, J. Opt. Commun. 8, 49 (1987)

[7] A W Warner, M Onoe and G A Coquin, J. Acoust. Soc. Am. 42, 1223 (1967)

[8] G W Farnell, IEEE Trans. Sonics, Ultrason, Ferroelect. Freq. Control SU17 229 (1970)

[9] A K Ghatak and K Thyagarajan, Optical electronics (Cambridge University Press, London, 1989) ch. 14, p. 427

[10] C S Tsai, M A Alhaider, L T Nguyen and B Kim, Proc. IEEE 64, 318 (1976)

[11] S Mc Meekin and R M De la Rue, Electron. Lett. 25, 853 (1989)

[12] A Loni, G Hay, R M De la Rue and J M Winfield, IEEE J. Lightwave Technol. 7,911 (1989) 